\title{
A "COBOUNDARY" THEOREM FOR SUMS OF RANDOM VARIABLES TAKING THEIR VALUES IN A BANACH SPACE
}

\author{
Richard C. BRADLEY
}

Klaus Schmidt proved that if a strictly stationary sequence of (say) real-valued random variables is such that the family of distributions of its partial sums is tight, then that sequence is a "coboundary". Here Schmidt's result is extended to some (not necessarily stationary) sequences of random variables taking their values in a separable real Banach space.

\section{Introduction.}

For a given metric space $(S, d)$, let us use the term "standard $\sigma$-field" to denote the $\sigma$-field $\mathcal{S}$ of subsets of $S$ generated by the open balls (in the metric $d$ ). A function $f: S \times S \times S \times \ldots \rightarrow S$ is "measurable" (with respect to $\mathcal{S}$ ) if for every set $A \in \mathcal{S}$ one has that $f^{-1}(A)$ is a member of $\mathcal{S} \times \mathcal{S} \times \mathcal{S} \times \ldots$, the product $\sigma$-field on $S \times S \times S \times \ldots$.

Suppose $B$ is a separable real Banach space. Suppose $(\Omega, \mathcal{F}, P)$ is a probability space. A " $B$-valued random variable" is of course a function $X: \Omega \rightarrow B$ such that, letting $\mathcal{B}$ denote the standard $\sigma$-field on $B$, for every set $A \in \mathcal{B}$ one has that $X^{-1}(A) \in \mathcal{F}$.

Given a sequence $\left(X_{k}, k \in \mathbb{Z}\right)$ of $B$-valued random variables, for any pair of integers $J \leq L$ we shall denote the "partial sum"

$$
S(J, L):=\sum_{k=J}^{L} X_{k}
$$

(a $B$-valued random variable).

The purpose of this paper is to prove the following theorem:

Theorem 1.1. $\quad$ Suppose $B$ is a separable real Banach space. Suppose $\left(X_{k}, k \in \mathbb{Z}\right)$ is a sequence of B-valued random variables such that for each $k \in \mathbb{Z}$, the family of distributions of the random variables $(S(k, m), m=$ $k, k+1, k+2, \ldots)$ is tight. Then there exists a measurable function $f$ : 
$B \times B \times B \times \ldots \rightarrow B$ such that, defining for each $k \in \mathbb{Z}$ the B-valued random variable

$$
Y_{k}:=f\left(X_{k}, X_{k+1}, X_{k+2}, \ldots\right),
$$

one has that

$$
\forall k \in \mathbb{Z}, X_{k}=Y_{k}-Y_{k+1} \text { a.s. }
$$

In the literature on ergodic theory, (1.1) would be described by saying that the sequence $\left(X_{k}\right)$ is a "coboundary."

In the case where $B=\mathbb{R}^{n}$ (say with the Euclidean norm) for some positive integer $n$, and the sequence $\left(X_{k}\right)$ is strictly stationary, Theorem 1.1 is due to K. Schmidt [10, Theorem 11.8]. With a modification of Schmidt's argument, the author [3] showed that for real-valued random variables, Theorem 1.1 holds in its present form (without the assumption of stationarity). The main purpose of that note was to call attention to Schmidt's result (which was not well known) and to try to make it more transparent. Here in Theorem 1.1 we extend Schmidt's result (and its extension in [3]) to $B$-valued random variables (again without the assumption of stationarity). The proof here will be a modification of that in [3] (which in turn was a modification of Schmidt's argument). Some parts of the argument in [3] will be adapted directly here. However, because of the extensive changes and additions that are needed, the proof here will be given in full. In a related paper, the author [4] proved an analog of Schmidt's result for products of some random matrices.

In the case of a weakly stationary sequence $\left(X_{k}, k \in \mathbb{Z}\right)$ of real-valued, mean zero, square integrable random variables, Leonov [8] had earlier proved an analog of Schmidt's result under the assumption that $\sup _{n \geq 1} \operatorname{Var} S(1, n)<$ $\infty$. (There is more to Leonov's result than that; for details, see [8] or Ibragimov and Linnik [7, p. 323, Theorem 18.2.2]. Further research in this direction was done by Gordin [6].)

Remark 1.2. Using standard results in e.g. Billingsley [2], it is easy to verify that in the statement of Theorem 1.1, the assumption "for each $k \in \mathbb{Z}$, the family ... is tight" is equivalent to "for some $k \in \mathbb{Z}$, the family ... is tight". Also, in Theorem 1.1, the family of distributions of the random variables $\left(Y_{k}, k \in \mathbb{Z}\right)$ is tight if and only if the family of distributions of $(S(k, m), k \in \mathbb{Z}, m \geq k)$ is tight. Also, if the sequence $\left(X_{k}\right)$ is strictly stationary then so is $\left(Y_{k}\right)$, and if $\left(X_{k}\right)$ is ergodic then so is $\left(Y_{k}\right)$. In Theorem 1.1, the restriction to "real" Banach spaces is of course only a matter of style; any complex Banach space is also a real one (with the scalars restricted). 
Theorem 1.1 will be proved first in Section 2 in the special case where $B=C[0,1]$ (real continuous functions), and then from that special case it will be derived in Section 3 for general separable real Banach spaces.

In what follows, the indicator function of an event $A$ will be denoted $I(A)$.

\section{Proof of Theorem 1.1 for the case $B=C[0,1]$.}

In this section we shall prove Theorem 1.1 in the special case where the (real separable) Banach space is $C[0,1]$, the space of real continuous functions on the closed unit interval $[0,1]$ with the usual sup norm

$$
\|x\|_{\infty}:=\sup _{t \in[0,1]}|x(t)| .
$$

For each $\delta>0$, define the usual "modulus of continuity" function $w_{\delta}$ : $C[0,1] \rightarrow[0, \infty)$ by

$$
\begin{aligned}
w_{\delta}(x):= & \sup |x(s)-x(t)| \\
& s, t \in[0,1] \\
& |s-t| \leq \delta .
\end{aligned}
$$

Remark 2.1. In what follows, the following three elementary facts will be used freely:

(a) The function $x \mapsto\|x\|_{\infty}$ is a continuous, hence measurable function from $C[0,1]$ to $[0, \infty)$.

(b) For each $t \in[0,1]$, the function $x \mapsto x(t)$ is a continuous, hence measurable function from $C[0,1]$ to $\mathbb{R}$.

(c) For each $\delta>0$, the function $w_{\delta}$ in (2.2) is a continuous, hence measurable function from $C[0,1]$ to $[0, \infty)$.

The following lemma is well known; for example it is an easy corollary of Billingsley [2, p. 55, Theorem 8.2].

Lemma 2.2. Suppose $\left(V_{1}, V_{2}, V_{3}, \ldots\right)$ is a sequence of $C[0,1]$-valued random variables. The family of distributions of these random variables $\left(V_{1}, V_{2}, V_{3}, \ldots\right)$ is tight if and only if the following two conditions hold:

(a) The family of distributions of the real-valued non-negative random variables $\left(\left\|V_{1}\right\|_{\infty},\left\|V_{2}\right\|_{\infty},\left\|V_{3}\right\|_{\infty}, \ldots\right)$ is tight.

(b) For every $\varepsilon>0$, there exists $\delta=\delta(\varepsilon)>0$ such that for every $n \in$ $\mathbb{N}, P\left(w_{\delta}\left(V_{n}\right) \geq \varepsilon\right) \leq \varepsilon$.

Now we are ready to begin the proof of Theorem 1.1 for the Banach space $C[0,1]$. Assume that $(\Omega, \mathcal{F}, P)$ is a probability space, and that $\left(X_{k}, k \in \mathbb{Z}\right)$ is 
a sequence of $C[0,1]$-valued random variables on this probability space, such that the hypothesis of Theorem 1.1 is satisfied. Without loss of generality, we assume that for every $k \in \mathbb{Z}$, every $\omega \in \Omega, X_{k}(\omega):=\left(X_{k}(t)(\omega), t \in[0,1]\right)$ is an element of $C[0,1]$. (This assumption will help in keeping track of "bad" null-sets later on.)

The proof will be structured as follows: Preliminary work is done from Definition 2.3 through Lemma 2.22, the random variables $Y_{k}$ are formulated in Definition 2.23, in Lemma 2.24 it is shown that $X_{k}=Y_{k}-Y_{k+1}$ a.s., the (measurable) function $f$ is formulated in Definition 2.25, and finally in Step 2.26 it is shown that $Y_{k}=f\left(X_{k}, X_{k+1}, X_{k+2}, \ldots\right)$.

Definition 2.3. For each $k \in \mathbb{Z}$, each $n \geq 1$, each $r \in \mathbb{R}$, each $t \in[0,1]$, define the (real) random variable

$$
V(k, n, r, t):=\frac{1}{n} \sum_{h=0}^{n-1} I(S(k, k+h)(t) \leq r) .
$$

For each $k \in \mathbb{Z}$, each $n \geq 1$, each $r>0$, define the (real) random variable

$$
V^{*}(k, n, r):=\frac{1}{n} \sum_{h=0}^{n-1} I\left(\|S(k, k+h)\|_{\infty} \leq r\right) .
$$

For each $k \in \mathbb{Z}$, each $n \geq 1$, each $\delta>0$, each $\varepsilon>0$, define the (real) random variable

$$
V^{* *}(k, n, \delta, \varepsilon):=\frac{1}{n} \sum_{h=0}^{n-1} I\left(w_{\delta}(S(k, k+h)) \geq \varepsilon\right) .
$$

\section{Remark 2.4.}

(a) Note that (at every $\omega \in \Omega$ ), all of these random variables $V(k, n, r, t)$, $V^{*}(k, n, r), V^{* *}(k, n, \delta, \varepsilon)$ take their values in the closed unit interval $[0,1]$.

(b) Let us digress a little, to mention a useful fact: If $\left(W_{n}^{(\lambda)}, n \in \mathbb{N}, \lambda \in \Lambda\right.$ ) is an array of random variables taking their values in $[0,1]$, with $\Lambda$ being a countable index set, then there exists a strictly increasing sequence of positive integers $m(1), m(2), m(3), \ldots$ such that for all $\lambda \in \Lambda, \quad n^{-1} \sum_{j=1}^{n} W_{m(j)}^{(\lambda)}$ converges a.s. (to a r.v. which depends on $\lambda$ ) as $n \rightarrow \infty$. To prove this fact, one first applies repeatedly the Komlós strong law of large numbers (see e.g. the extension of it given by Berkes [1, Theorem 2]), and then one uses a simple Cantor diagonalization argument. (The details of this proof are left to the reader.) This fact will be used freely in what follows; in particular, it justifies the next definition: 
Definition 2.5. Let $\mathbb{Q}$ denote the set of all rational numbers. Let $\mathbb{Q}_{(0, \infty)}$ denote the set of all positive rational numbers. Let $\mathbb{Q}_{[0,1]}$ denote the set of all rational numbers in $[0,1]$.

Let $\left(m_{1}, m_{2}, m_{3}, \ldots\right)$ be a strictly increasing sequence of positive integers such that the following three statements hold:

(1) For each $k \in \mathbb{Z}$, each $r \in \mathbb{Q}$, each $t \in \mathbb{Q}_{[0,1]}$,

$$
\frac{1}{n} \sum_{j=1}^{n} V\left(k, m_{j}, r, t\right) \text { converges a.s. as } n \rightarrow \infty \text {. }
$$

(2) For each $k \in \mathbb{Z}$, each $r \in \mathbb{Q}_{(0, \infty)}$,

$$
\frac{1}{n} \sum_{j=1}^{n} V^{*}\left(k, m_{j}, r\right) \text { converges a.s. as } n \rightarrow \infty \text {. }
$$

(3) For each $k \in \mathbb{Z}$, each $\delta \in \mathbb{Q}_{(0, \infty)}$, each $\varepsilon \in \mathbb{Q}_{(0, \infty)}$,

$$
\frac{1}{n} \sum_{j=1}^{n} V^{* *}\left(k, m_{j}, \delta, \varepsilon\right) \text { converges a.s. as } n \rightarrow \infty \text {. }
$$

Definition 2.6. For each $k \in \mathbb{Z}$, each $r \in \mathbb{R}$, each $t \in[0,1]$, define the (real) random variable

$$
Z(k, r, t):=\varlimsup_{n \rightarrow \infty} \frac{1}{n} \sum_{j=1}^{n} V\left(k, m_{j}, r, t\right) .
$$

For each $k \in \mathbb{Z}$, each $r>0$, define the (real) random variable

$$
Z^{*}(k, r):=\varlimsup_{n \rightarrow \infty} \frac{1}{n} \sum_{j=1}^{n} V^{*}\left(k, m_{j}, r\right) .
$$

For each $k \in \mathbb{Z}$, each $\delta>0$, each $\varepsilon>0$, define the (real) random variable

$$
Z^{* *}(k, \delta, \varepsilon):=\varlimsup_{n \rightarrow \infty} \frac{1}{n} \sum_{j=1}^{n} V^{* *}\left(k, m_{j}, \delta, \varepsilon\right) .
$$

Functions such as $Z(k, r, t)$ played a role in Schmidt's [10] original argument, analogous to the role they will play here.

\section{Remark 2.7.}

(a) For every $\omega \in \Omega$, these random variables $Z(k, r, t), Z^{*}(k, r), Z^{* *}(k, \delta, \varepsilon)$ all take their values in the closed unit interval $[0,1]$. 
(b) For each $\omega \in \Omega$, each $k \in \mathbb{Z}$, each $t \in[0,1]$, the r.v. $Z(k, r, t)$ is nondecreasing as $r$ increases, by (2.3) and (2.9). (That is, for each $\omega \in \Omega$, each $k \in \mathbb{Z}$, each $t \in[0,1]$, the real number $Z(k, r, t)(\omega)$ is nondecreasing as $r$ increases.)

(c) Similarly, for each $\omega \in \Omega$ each $k \in \mathbb{Z}$, the r.v. $Z^{*}(k, r)$ is nondecreasing as $r$ increases, by (2.4) and (2.10).

(d) Similarly, for each $\omega \in \Omega$, each $k \in \mathbb{Z}$, each $\delta>0$, the r.v. $Z^{* *}(k, \delta, \varepsilon)$ is nonincreasing as $\varepsilon$ increases, by (2.5) and (2.11).

(e) When $r \in \mathbb{Q}\left(\right.$ or $\left.\mathbb{Q}_{(0, \infty)}\right), t \in \mathbb{Q}_{[0,1]}, \delta \in \mathbb{Q}_{(0, \infty)}, \varepsilon \in \mathbb{Q}_{(0, \infty)}$, the lim sup in each of (2.9), (2.10), and (2.11) is a.s. a limit.

Lemma 2.8. For each $k \in \mathbb{Z}$, each $r>0$, each $t \in[0,1]$, the following two statements hold:

(a) $Z^{*}(k, r) \leq Z(k, r, t) \quad($ at every $\omega \in \Omega)$.

(b) For every $\gamma>0, Z^{*}(k, r) \leq 1-Z(k,-r-\gamma, t)$ a.s.

Proof. (a) For each $h \geq 0$ (and each $\omega \in \Omega$ ), one clearly has

$$
I\left(\|S(k, k+h)\|_{\infty} \leq r\right) \leq I(S(k, k+h)(t) \leq r) .
$$

Hence by (2.3) and (2.4), for each $n \geq 1$ (and each $\omega \in \Omega$ ),

$$
V^{*}(k, n, r) \leq V(k, n, r, t) .
$$

Hence (a) holds by (2.9) and (2.10).

(b) Suppose $\gamma>0$. Let $q \in \mathbb{Q}$ be such that $r<q<r+\gamma$. For each $h \geq 0$,

$$
\begin{aligned}
I\left(\|S(k, k+h)\|_{\infty} \leq r\right) & \leq I\left(\|S(k, k+h)\|_{\infty} \leq q\right) \\
& \leq I(S(k, k+h)(t)>-r-\gamma) \\
& =1-I(S(k, k+h)(t) \leq-r-\gamma) .
\end{aligned}
$$

Hence by (2.3) and (2.4), for each $n \geq 1$,

$$
V^{*}(k, n, r) \leq V^{*}(k, n, q) \leq 1-V(k, n,-r-\gamma, t) .
$$

Hence by (2.9), (2.10), and Remark 2.7(c)(e), one has that with probability 1 ,

$$
\begin{aligned}
Z^{*}(k, r) & \leq Z^{*}(k, q) \\
& =\lim _{n \rightarrow \infty} \frac{1}{n} \sum_{j=1}^{n} V^{*}\left(k, m_{j}, q\right)
\end{aligned}
$$




$$
\begin{aligned}
& \leq \liminf _{n \rightarrow \infty} \frac{1}{n} \sum_{j=1}^{n}\left[1-V\left(k, m_{j},-r-\gamma, t\right)\right] \\
& =1-\lim _{n \rightarrow \infty} \sup _{n} \frac{1}{n} \sum_{j=1}^{n} V\left(k, m_{j},-r-\gamma, t\right) \\
& =1-Z(k,-r-\gamma, t) .
\end{aligned}
$$

Thus (b) holds.

Lemma 2.9. Suppose $k \in \mathbb{Z}, r \in \mathbb{R}, \delta>0$, and $\varepsilon>0$. Suppose $s$ and $t$ are elements of $[0,1]$ such that $|s-t| \leq \delta$. Then (at every $\omega \in \Omega) Z(k, r, s) \leq$ $Z(k, r+\varepsilon, t)+Z^{* *}(k, \delta, \varepsilon)$.

Proof. By an elementary argument, for each $h \geq 0$ (and each $\omega \in \Omega$ ),

$$
\begin{aligned}
I(S(k, k+h)(s) \leq r) \leq & I(S(k, k+h)(t) \leq r+\varepsilon) \\
& +I\left(w_{\delta}(S(k, k+h)) \geq \varepsilon\right) .
\end{aligned}
$$

Hence by (2.3) and (2.5), for each $n \geq 1$ (and each $\omega \in \Omega$ ),

$$
V(k, n, r, s) \leq V(k, n, r+\varepsilon, t)+V^{* *}(k, n, \delta, \varepsilon) .
$$

Hence by (2.9) and (2.11), Lemma 2.9 holds.

Lemma 2.10. For each $k \in \mathbb{Z}$, each $t \in \mathbb{Q}_{[0,1]}$, the following two statements hold:

(a) $Z(k, r, t) \rightarrow 0 \quad$ a.s. $\quad$ as $\quad r \rightarrow-\infty$.

(b) $Z(k, r, t) \rightarrow 1 \quad$ a.s. as $r \rightarrow \infty$.

Proof. Let $k \in \mathbb{Z}$ and $t \in \mathbb{Q}_{[0,1]}$ be arbitrary but fixed. Our task is to verify (a) and (b) in Lemma 2.10 for this pair $k, t$.

Applying Lemma 2.2 and the hypothesis of Theorem 1.1, for each $\ell=$ $1,2,3, \ldots$ let $r_{\ell} \in \mathbb{Q}_{(0, \infty)}$ be such that

$$
\forall h \geq 0, P\left(-r_{\ell}<S(k, k+h)(t) \leq r_{\ell}\right) \geq 1-4^{-\ell} .
$$

That is, for each $\ell \geq 1$, each $h \geq 0$,

$$
E I\left(S(k, k+h)(t) \leq r_{\ell}\right)-E I\left(S(k, k+h)(t) \leq-r_{\ell}\right) \geq 1-4^{-\ell} .
$$

Consequently, for all $\ell \geq 1$, all $n \geq 1$,

$$
E V\left(k, n, r_{\ell}, t\right)-E V\left(k, n,-r_{\ell}, t\right) \geq 1-4^{-\ell} .
$$


Hence by Remarks 2.4(a) and 2.7(e) and dominated convergence,

$$
E Z\left(k, r_{\ell}, t\right)-E Z\left(k,-r_{\ell}, t\right) \geq 1-4^{-\ell} .
$$

Hence by Remark 2.7(a),

$$
E Z\left(k,-r_{\ell}, t\right) \leq 4^{-\ell} \text { and } 1-E Z\left(k, r_{\ell}, t\right) \leq 4^{-\ell} .
$$

Let us first verify (b) in Lemma 2.10 (for our fixed $k, t)$. For each $\ell \geq 1$,

$$
\begin{aligned}
P\left(Z\left(k, r_{\ell}, t\right) \leq 1-2^{-\ell}\right) & =P\left(1-Z\left(k, r_{\ell}, t\right) \geq 2^{-\ell}\right) \\
& \leq E\left(1-Z\left(k, r_{\ell}, t\right)\right) / 2^{-\ell} \\
& \leq 4^{-\ell} / 2^{-\ell}=2^{-\ell} .
\end{aligned}
$$

By the Borel-Cantelli Lemma,

$$
P\left(Z\left(k, r_{\ell}, t\right) \leq 1-2^{-\ell} \text { for infinitely many } \ell \geq 1\right)=0 .
$$

Lemma 2.10(b) follows (for our fixed $k, t$ ).

By a similar argument, $P\left(Z\left(k,-r_{\ell}, t\right) \geq 2^{-\ell}\right) \leq 2^{-\ell}$ for each $\ell \geq 1$, and Lemma 2.10(a) follows from the Borel-Cantelli Lemma and Remark 2.7(b). This completes the proof of Lemma 2.10.

Lemma 2.11. For each $k \in \mathbb{Z}$, one has that

$$
Z^{*}(k, r) \rightarrow 1 \quad \text { a.s. as } r \rightarrow \infty .
$$

Proof. Let $k \in \mathbb{Z}$ be arbitrary but fixed. Applying Lemma 2.2 and the hypothesis of Theorem 1.1, for each $\ell=1,2,3, \ldots$ let $r_{\ell} \in \mathbb{Q}_{(0, \infty)}$ be such that

$$
\forall h \geq 0, P\left(\|S(k, k+h)\|_{\infty} \leq r_{\ell}\right) \geq 1-4^{-\ell} .
$$

Then $E V^{*}\left(k, n, r_{\ell}\right) \geq 1-4^{-\ell}$ for all $\ell \geq 1$, all $n \geq 1$; and by Remarks 2.4(a) and 2.7(e) and dominated convergence, $E Z^{*}\left(k, r_{\ell}\right) \geq 1-4^{-\ell}$ for all $\ell \geq 1$. Now Eqns. (2.12) and (2.13) hold with $Z^{*}\left(k, r_{\ell}\right)$ in place of $Z\left(k, r_{\ell}, t\right)$; and Lemma 2.11 follows.

Lemma 2.12. For each $k \in \mathbb{Z}$, each $\varepsilon>0$, each $\gamma>0$, there exists $\delta>0$ such that

$$
P\left(Z^{* *}(k, \delta, \varepsilon) \geq \gamma\right) \leq \gamma
$$

Proof. Let $k \in \mathbb{Z}, \varepsilon>0$, and $\gamma>0$ be arbitrary but fixed. Decreasing $\varepsilon$ if necessary, we assume without loss of generality that $\varepsilon \in \mathbb{Q}_{(0, \infty)}$. 
Applying Lemma 2.2 and the hypothesis of Theorem 1.1, let $\delta \in \mathbb{Q}_{(0, \infty)}$ be such that

$$
\forall h \geq 0, P\left(w_{\delta}(S(k, k+h)) \geq \varepsilon\right) \leq \gamma^{2} .
$$

Then for all $n \geq 1, E V^{* *}(k, n, \delta, \varepsilon) \leq \gamma^{2}$, and by Remarks 2.4(a) and 2.7(e) and dominated convergence, $E Z^{* *}(k, \delta, \varepsilon) \leq \gamma^{2}$. Hence $P\left(Z^{* *}(k, \delta, \varepsilon) \geq \gamma\right) \leq$ $\gamma^{2} / \gamma=\gamma$. This completes the proof of the lemma.

\section{Definition 2.13.}

(a) In what follows, an "extended" random variable will mean a measurable function from $\Omega$ to $\mathbb{R} \cup\{-\infty,+\infty\}$.

(b) For the empty set $\phi$, define the "infimum" by inf $\phi:=+\infty$. For a (nonempty) set $S$ of real numbers which is unbounded below, of course define the "infimum" by inf $S:=-\infty$.

(c) For each $k \in \mathbb{Z}$, each $u \in(0,1)$, each $t \in[0,1]$, define the extended random variable $Q(k, u, t)$ as follows: For all $\omega \in \Omega$,

$$
Q(k, u, t)(\omega):=\inf \{r \in \mathbb{R}: Z(k, r, t)(\omega) \geq u\} .
$$

For each $k \in \mathbb{Z}$, each $u \in(0,1)$, define the (nonnegative) extended random variable $Q^{*}(k, u)$ as follows: For all $\omega \in \Omega$,

$$
Q^{*}(k, u)(\omega):=\inf \left\{r>0: Z^{*}(k, r)(\omega) \geq u\right\} .
$$

In (2.14) and (2.15) the conventions in (a) and (b) are used where necessary. The fact that $Q(k, u, t)$ and $Q^{*}(k, u)$ are measurable functions from $\Omega$ to $\mathbb{R} \cup\{-\infty,+\infty\}$, is an elementary consequence of Remarks $2.7(\mathrm{~b})(\mathrm{c})$.

\section{Remark 2.14.}

(a) For each $\omega \in \Omega$, each $k \in \mathbb{Z}$, each $t \in \mathbb{R}$, the extended r.v. $Q(k, u, t)$ is nondecreasing (in $\{-\infty\} \cup \mathbb{R} \cup\{\infty\}$ ) as $u$ increases in $(0,1)$, by Remark $2.7(\mathrm{~b})$.

(b) For each $\omega \in \Omega$, each $k \in \mathbb{Z}$, the extended r.v. $Q^{*}(k, u)$ is nondecreasing (in $[0, \infty])$ as $u$ increases in $(0,1)$, by Remark $2.7(\mathrm{c})$.

(c) For each $k \in \mathbb{Z}$, each $t \in \mathbb{Q}_{[0,1]}, P(-\infty<Q(k, u, t)<\infty \quad \forall u \in$ $(0,1))=1$ by Lemma 2.10 .

(d) For each $k \in \mathbb{Z}, P\left(0 \leq Q^{*}(k, u)<\infty \quad \forall u \in(0,1)\right)=1$ by Lemma 2.11 .

Lemma 2.15. For each $k \in \mathbb{Z}$, each $t \in[0,1]$, one has that

$$
P\left(|Q(k, u, t)| \leq Q^{*}(k, .8) \forall u \in[.3, .8]\right)=1 .
$$


Proof. Let $k \in \mathbb{Z}$ and $t \in[0,1]$ be arbitrary but fixed. By Remark (2.14)(a), it suffices to prove that

$$
Q(k, .8, t) \leq Q^{*}(k, .8) \text { a.s. }
$$

and

$$
-Q(k, .3, t) \leq Q^{*}(k, .8) \text { a.s. }
$$

For each $\omega \in \Omega$, by Lemma 2.8(a),

$$
\left\{r>0: Z^{*}(k, r)(\omega) \geq .8\right\} \subset\{r \in \mathbb{R}: Z(k, r, t)(\omega) \geq .8\} .
$$

Hence (2.16) holds by Definition 2.13(c). Now we need to prove (2.17).

Let $\Omega_{0}$ denote the set of all $\omega \in \Omega$ such that $Q^{*}(k, .8)(\omega)<\infty$ and for every pair of numbers $r \in \mathbb{Q}_{(0, \infty)}$ and $\gamma \in \mathbb{Q}_{(0, \infty)}$, one has that

$$
Z^{*}(k, r)(\omega) \leq 1-Z(k,-r-\gamma, t)(\omega)
$$

By Lemma 2.8(b), $P\left(\Omega_{0}\right)=1$.

Let $\omega \in \Omega_{0}$ be arbitrary but fixed. Let $\gamma \in \mathbb{Q}_{(0, \infty)}$ be arbitrary but fixed. To prove (2.17), it suffices to prove for this $\omega$ and this $\gamma$ that

$$
-2 \gamma-Q(k, .3, t)(\omega) \leq Q^{*}(k, .8)(\omega)
$$

Denote the nonnegative number $q:=Q^{*}(k, .8)(\omega)$. Let $q^{\prime} \in \mathbb{Q}$ be such that $q^{\prime}<q<q^{\prime}+\gamma$. (Note that $q^{\prime}+\gamma>0$.) Now $Z^{*}\left(k, q^{\prime}+\gamma\right)(\omega) \geq .8$ by Definition 2.13(c) and Remark 2.7(c). Hence $1-Z\left(k,-q^{\prime}-2 \gamma, t\right)(\omega) \geq .8$ by (2.18) and our assumption that $\omega \in \Omega_{0}$. Hence $Z\left(k,-q^{\prime}-2 \gamma, t\right)(\omega) \leq .2$. Hence $-q^{\prime}-2 \gamma \leq Q(k, .3, t)(\omega)$ by Definition 2.13(c) and Remark 2.7(b). Hence $-q-2 \gamma \leq Q(k, .3, t)(\omega)$. Thus (2.19) holds. This completes the proof of (2.17) and of Lemma 2.15.

Remark 2.16. Let us digress for a moment to mention an elementary fact of calculus that will simplify some technicalities in what follows: If $a<b$ are real numbers and $g:[a, b] \rightarrow \mathbb{R}$ is a monotonic (hence bounded) function, then

$$
\int_{a}^{b} g(u) d u=\lim _{n \rightarrow \infty} \frac{b-a}{n} \sum_{j=0}^{n-1} g\left(a+\frac{j}{n}(b-a)\right) .
$$


Definition 2.17. For each $k \in \mathbb{Z}$, each $t \in \mathbb{Q}_{[0,1]}$, define the (real) random variable $W(k, t)$ as follows: For $\omega \in \Omega$,

$$
W(k, t)(\omega):=\left\{\begin{array}{lll}
5 \cdot \int_{.4}^{.6} Q(k, u, t)(\omega) d u & \text { if }-\infty<Q(k, u, t)(\omega)<\infty \\
0 & \forall u \in(0,1), \\
& \text { otherwise. }
\end{array}\right.
$$

Remark 2.18. Let us briefly justify Definition 2.17. By Remark 2.14(a), for any $\omega \in \Omega$ such that $-\infty<Q(k, u, t)(\omega)<\infty \quad \forall u \in(0,1)$, the integral in (2.20) is well defined as a real number. By Remarks 2.14(c) and 2.16, one has that with probability 1 ,

$$
\begin{aligned}
W(k, t) & =5 \cdot \int_{.4}^{.6} Q(k, u, t) d u \\
& =\lim _{n \rightarrow \infty}\left[\frac{1}{n} \sum_{j=0}^{n-1} Q\left(k,(.4)+\frac{j}{n}(.2), t\right)\right] .
\end{aligned}
$$

In particular, this gives a simple way of confirming that the function $W(k, t)$ defined on $\Omega$ in Definition 2.17 really is a random variable (i.e. real, measurable).

Lemma 2.19. For each $k \in \mathbb{Z}$, each $t \in \mathbb{Q}_{[0,1]}$, one has that

$$
W(k, t)=X_{k}(t)+W(k+1, t) \quad \text { a.s. }
$$

Before proving Lemma 2.19, let us motivate it. Our task later on will be to define for each $k \in \mathbb{Z}$ a $C[0,1]$-valued r.v. $Y_{k}$ satisfying $Y_{k}(t)=W(k, t)$ a.s. for $t \in \mathbb{Q}_{[0,1]}$. This will be justified by Lemmas 2.20 and 2.22 below. We shall then have the desired equation $Y_{k}=X_{k}+Y_{k+1}$ (i.e. $X_{k}=Y_{k}-Y_{k+1}$ ) a.s.

Proof. Let $k \in \mathbb{Z}$ and $t \in \mathbb{Q}_{[0,1]}$ be arbitrary but fixed.

For each $r \in \mathbb{R}$, each $n \geq 1$,

$$
|V(k, n, r, t)-V(k, n+1, r, t)|
$$

$$
=\left|-\frac{1}{n+1} I(S(k, k+n)(t) \leq r)+\left(\frac{1}{n}-\frac{1}{n+1}\right) \sum_{h=0}^{n-1} I(S(k, k+h)(t) \leq r)\right|
$$


$\leq 2 / n$.

For each $\omega \in \Omega$, each $r \in \mathbb{R}$, each $n \geq 1$

$$
\begin{aligned}
& \sum_{h=0}^{n} I\left(S(k, k+h)(t)(\omega) \leq r+X_{k}(t)(\omega)\right) \\
& =I\left(X_{k}(t)(\omega) \leq r+X_{k}(t)(\omega)\right) \\
& \quad+\sum_{h=0}^{n-1} I\left(S(k, k+1+h)(t)(\omega) \leq r+X_{k}(t)(\omega)\right) \\
& =I\left(X_{k}(t)(\omega) \leq r+X_{k}(t)(\omega)\right) \\
& \quad+\sum_{h=0}^{n-1} I(S(k+1, k+1+h)(t)(\omega) \leq r)
\end{aligned}
$$

and hence

$$
\begin{aligned}
\left|V\left(k, n+1, r+X_{k}(t)(\omega), t\right)(\omega)-V(k+1, n, r, t)(\omega)\right| \\
=\mid \frac{1}{n+1} I\left(X_{k}(t)(\omega) \leq r+X_{k}(t)(\omega)\right) \\
\quad+\left(\frac{1}{n+1}-\frac{1}{n}\right) \sum_{h=0}^{n-1} I(S(k+1, k+1+h)(t)(\omega) \leq r) \mid \\
\leq 2 / n .
\end{aligned}
$$

By combining (2.22) (with $r$ replaced by $\left.r+X_{k}(t)(\omega)\right)$ with (2.23), we have that for each $\omega \in \Omega$, each $r \in \mathbb{R}$, each $n \geq 1$,

$$
\left|V\left(k, n, r+X_{k}(t)(\omega), t\right)(\omega)-V(k+1, n, r, t)(\omega)\right| \leq \frac{4}{n} .
$$

Hence for each $\omega \in \Omega$, each $r \in \mathbb{R}$, each $n \geq 1$,

$$
\begin{aligned}
& \left|\frac{1}{n} \sum_{j=1}^{n} V\left(k, m_{j}, r+X_{k}(t)(\omega), t\right)(\omega)-\frac{1}{n} \sum_{j=1}^{n} V\left(k+1, m_{j}, r, t\right)(\omega)\right| \\
& \leq \frac{1}{n} \sum_{j=1}^{n} 4 / m_{j} .
\end{aligned}
$$

Hence by Definition 2.6 and Toeplitz' Lemma, for each $\omega \in \Omega$, each $r \in \mathbb{R}$,

$$
Z\left(k, r+X_{k}(t)(\omega), t\right)(\omega)=Z(k+1, r, t)(\omega) .
$$


Now let $\Omega_{0}$ denote the set of all $\omega \in \Omega$ such that $\forall j \in \mathbb{Z}, \forall u \in$ $(0,1),-\infty<Q(j, u, t)(\omega)<\infty$. By Remark 2.14(c),

$$
P\left(\Omega_{0}\right)=1
$$

For every $\omega \in \Omega_{0}$, every $u \in(0,1)$, one has that

$$
Q(k, u, t)(\omega)=X_{k}(t)(\omega)+Q(k+1, u, t)(\omega) .
$$

This can be shown as follows: Denote $a:=Q(k, u, t)(\omega)$ and $b:=Q(k+$ $1, u, t)(\omega)$. For each $r<b$, one has that

$$
Z\left(k, r+X_{k}(t)(\omega), t\right)(\omega)=Z(k+1, r, t)(\omega)<u
$$

by (2.24) and (2.14), and hence $r+X_{k}(t)(\omega) \leq a$ by (2.14) (and Remark $2.14(\mathrm{a}))$. Hence $b+X_{k}(t)(\omega) \leq a$. For each $r>b$, one has that

$$
Z\left(k, r+X_{k}(t)(\omega), t\right)(\omega)=Z(k+1, r, t)(\omega) \geq u
$$

by (2.24) and (2.14), and hence $r+X_{k}(t)(\omega) \geq a$ by (2.14). Hence $b+$ $X_{k}(t)(\omega) \geq a$. Hence $b+X_{k}(t)(\omega)=a$. That is, (2.26) holds.

Hence for each $\omega \in \Omega_{0}, \quad W(k, t)(\omega)=X_{k}(t)(\omega)+W(k+1, t)(\omega)$ by (2.26), Definition 2.17, and a simple calculation. Hence by (2.25), Lemma 2.19 holds.

Lemma 2.20. Suppose $k \in \mathbb{Z}, \delta>0$, and $\varepsilon>0$. Suppose $s$ and $t$ are elements of $\mathbb{Q}_{[0,1]}$ such that $|s-t| \leq \delta$. Then for a.e. $\omega \in\left\{Z^{* *}(k, \delta, \varepsilon) \leq .1\right\}$, one has that

$$
|W(k, s)(\omega)-W(k, t)(\omega)| \leq \varepsilon+10 \cdot Z^{* *}(k, \delta, \varepsilon)(\omega) \cdot Q^{*}(k, .8)(\omega)
$$

(Here of course the notation "a.e." means "almost every" with respect to the given probability measure $P$ on $(\Omega, \mathcal{F})$. If $P\left(Z^{* *}(k, \delta, \varepsilon) \leq .1\right)=0$, then Lemma 2.20 is taken as "true by default".)

Proof. Let $k, \delta, \varepsilon, s$, and $t$ be as in the hypothesis of Lemma 2.20. Let $\Omega_{0}$ denote the set of all $\omega \in \Omega$ such that

(i) $-\infty<Q(k, u, s)(\omega)<\infty$ and $-\infty<Q(k, u, t)(\omega)<\infty$ for all $u \in(0,1)$,

(ii) $Q^{*}(k, .8)(\omega)<\infty$, and

(iii) $|Q(k, u, s)(\omega)| \leq Q^{*}(k, .8)(\omega)$ and $|Q(k, u, t)(\omega)| \leq Q^{*}(k, .8)(\omega)$ for all $u \in[.3, .8]$. 
By Remark 2.14(c)(d) and Lemma 2.15, one has that $P\left(\Omega_{0}\right)=1$. Let $\omega \in \Omega_{0} \cap\left\{Z^{* *}(k, \delta, \varepsilon) \leq .1\right\}$ be arbitrary but fixed. (If no such $\omega$ exists, then Lemma 2.20 is trivial; we assume such an $\omega$ exists.) To prove Lemma 2.20 , it suffices to prove for this $\omega$ that

$$
W(k, t)(\omega)-W(k, s)(\omega) \leq[\text { r.h.s. of }(2.27)] .
$$

(For then (2.28) would also hold with $s$ and $t$ reversed, by the same argument, and (2.27) would follow.)

Define the number

$$
z:=Z^{* *}(k, \delta, \varepsilon)(\omega)
$$

Under our assumptions,

$$
0 \leq z \leq 1
$$

Claim A. Under the above assumptions, for each $u$ such that $z<u<1$ one has that $Q(k, u-z, t)(\omega)-\varepsilon \leq Q(k, u, s)(\omega)$.

Proof. Let $u \in(z, 1)$ and $\gamma>0$ be arbitrary but fixed. To prove Claim A, it suffices to prove that

$$
Q(k, u-z, t)(\omega)-\varepsilon \leq Q(k, u, s)(\omega)+\gamma .
$$

Define the real number $r_{0}:=Q(k, u, s)(\omega)$.

Then

$$
u \leq Z\left(k, r_{0}+\gamma, s\right)(\omega) \leq Z\left(k, r_{0}+\gamma+\varepsilon, t\right)(\omega)+z
$$

by Definition 2.13(c), Remark 2.7(b), (2.29), and Lemma 2.9. That is, $u-z \leq$ $Z\left(k, r_{0}+\gamma+\varepsilon, t\right)(\omega)$. Hence

$$
Q(k, u-z, t)(\omega) \leq r_{0}+\gamma+\varepsilon,=Q(k, u, s)(\omega)+\gamma+\varepsilon
$$

by Definition 2.13(c). Thus (2.31) holds. This completes the proof of Claim A.

Now we are ready to finish the proof of (2.28). By Definition 2.17, Eqn. (2.30), Claim A, and the various properties of $\Omega_{0}$ (recall our assumption $\omega \in \Omega_{0}$ ), one has that

$$
W(k, s)(\omega)=5 \cdot \int_{.4}^{.6} Q(k, u, s)(\omega) d u
$$




$$
\begin{aligned}
\geq & 5 \cdot \int_{.4}^{.6}[Q(k, u-z, t)(\omega)] d u-\varepsilon \\
= & -\varepsilon+5 \cdot \int_{.4}^{.6} Q(k, u, t)(\omega) d u \\
& +5 \cdot \int_{.4-z}^{.4} Q(k, u, t)(\omega) d u \\
& -5 \cdot \int_{.6-z}^{.6} Q(k, u, t)(\omega) d u \\
\geq \quad & -\varepsilon+W(k, t)(\omega) \\
& -5 z \cdot Q^{*}(k, .8)(\omega)-5 z \cdot Q^{*}(k, .8)(\omega) .
\end{aligned}
$$

Thus (2.28) holds by (2.29). This completes the proof of Lemma 2.20.

Definition 2.21. For each $k \in \mathbb{Z}$, let $C_{k}$ denote the set of all $\omega \in \Omega$ such that $\left(W(k, t)(\omega), t \in \mathbb{Q}_{[0,1]}\right)$, as a function of $t$ from $\mathbb{Q}_{[0,1]}$ to $\mathbb{R}$, is uniformly continuous on $\mathbb{Q}_{[0,1]}$.

Lemma 2.22. For each $k \in \mathbb{Z}, P\left(C_{k}\right)=1$.

Proof. Let $k \in \mathbb{Z}$ be arbitrary but fixed. In the proof that $P\left(C_{k}\right)=1$, some of the notations will depend on this fixed value of $k$, but that dependence will be suppressed.

For each $\delta>0$, each positive integer $n$, let $A(\delta, n)$ denote the set of all $\omega \in \Omega$ such that the following holds: For every pair of numbers $s, t \in \mathbb{Q}_{[0,1]}$ such that $|s-t| \leq \delta$, one has that $|W(k, s)(\omega)-W(k, t)(\omega)| \leq 1 / n$.

Claim B. For each positive integer $n$, there exists $\delta>0$ such that $P(A(\delta, n)) \geq 1-2^{-n}$.

Before proving Claim B, let us first briefly explain why it implies Lemma 2.22. Suppose Claim B holds. For each $n \geq 1$ let $\delta_{n}>0$ be such that $P\left(A\left(\delta_{n}, n\right)\right) \geq 1-2^{-n}$. Let $B$ denote the set of all $\omega \in \Omega$ such that $\omega \notin$ $A\left(\delta_{n}, n\right)$ for at most finitely many $n$. By the Borel-Cantelli Lemma, $P(B)=$ 1. Clearly, for each $\omega \in B$, one has that $\left(W(k, t)(\omega), t \in \mathbb{Q}_{[0,1]}\right)$ is a uniformly continuous function of $t \in \mathbb{Q}_{[0,1]}$. Thus Lemma 2.22 follows from Claim B. Now we just need to prove Claim B.

Proof of Claim B. Let $n$ be an arbitrary fixed positive integer. It suffices to show for this $n$ that there exists $\delta>0$ such that

$$
P(A(\delta, n)) \geq 1-2^{-n}
$$


Applying Lemma 2.11, let $c$ be a positive number such that $P\left(Z^{*}(k, c) \geq\right.$ $.8) \geq 1-2^{-n-1}$. From Definition 2.13(c), one has that $P\left(Q^{*}(k, .8) \leq\right.$ c) $\geq 1-2^{-n-1}$. Next, applying Lemma 2.12 , let $\delta>0$ be such that

$$
P\left(Z^{* *}(k, \delta, 1 /(2 n)) \geq \min \{.1,1 /(20 n c)\}\right) \leq 2^{-n-1} .
$$

Denote by $D$ the event

$$
\left\{Q^{*}(k, .8) \leq c\right\} \cap\left\{Z^{* *}(k, \delta, 1 /(2 n)) \leq \min \{.1,1 /(20 n c)\}\right\} .
$$

Then $P(D) \geq 1-2^{-n}$.

Now by Lemma 2.20 , for a.e. $\omega \in D$, one has that for all $s, t \in \mathbb{Q}_{[0,1]}$ with $|s-t| \leq \delta$

$$
|W(k, s)(\omega)-W(k, t)(\omega)| \leq \frac{1}{2 n}+10 \cdot \frac{1}{20 n c} \cdot c=\frac{1}{n} .
$$

That is, a.e. $\omega \in D$ is an element of $A(\delta, n)$. Hence (2.32) holds. This completes the proof of Claim B and of Lemma 2.22.

Definition 2.23. For each $k \in \mathbb{Z}$, define the $C[0,1]$-valued r.v. $Y_{k}$ as follows: Referring to Definition 2.21, for each $\omega \in C_{k},\left(Y_{k}(t)(\omega), t \in[0,1]\right)$ is the unique element of $C[0,1]$ such that $Y_{k}(t)(\omega)=W(k, t)(\omega) \quad \forall t \in \mathbb{Q}_{[0,1]}$. For each $\omega \notin C_{k}, Y_{k}(t)(\omega):=0 \quad \forall t \in[0,1]$.

Lemma 2.24. For each $k \in \mathbb{Z}$, one has that $Y_{k}=X_{k}+Y_{k+1}$ a.s.

Proof. This follows easily from Definition 2.23 and Lemmas 2.19 and 2.22.

By Lemma 2.24, in order to complete the proof of Theorem 1.1 for $C[0,1]$ valued random variables, it suffices to produce a measurable function

$$
f: C[0,1] \times C[0,1] \times C[0,1] \times \ldots \rightarrow C[0,1]
$$

such that for each $k \in \mathbb{Z}$, each $\omega \in \Omega$,

$$
Y_{k}(\omega)=f\left(X_{k}(\omega), X_{k+1}(\omega), X_{k+2}(\omega), \ldots\right)
$$

Definition 2.25. In the definitions that follow, $x:=\left(x_{1}, x_{2}, x_{3}, \ldots\right)$ will denote a generic sequence of elements of $C[0,1]$. (This new use of the letter $x$ is different from that in Remark 2.1.) 
For parameters $n \geq 1, r \in \mathbb{R}$, and $t \in[0,1]$, each of the following is a measurable function from $C[0,1] \times C[0,1] \times C[0,1] \times \ldots$ into the closed unit interval $[0,1]$ :

$$
\begin{aligned}
I_{n, r, t}(x) & := \begin{cases}1 & \text { if } x_{1}(t)+\ldots+x_{n}(t) \leq r \\
0 & \text { otherwise }\end{cases} \\
v_{n, r, t}(x) & :=\frac{1}{n} \sum_{h=0}^{n-1} I_{h+1, r, t}(x) \\
z_{r, t}(x) & :=\varlimsup_{n \rightarrow \infty} \frac{1}{n} \sum_{j=1}^{n} v_{m(j), r, t}(x)
\end{aligned}
$$

where $m(j)$ denotes $m_{j}$ (from Definition 2.5). Note that for a given $t \in[0,1]$ and a given $x \in C[0,1] \times C[0,1] \times C[0,1], \ldots \quad z_{r, t}(x)$ is nondecreasing as $r$ increases in $\mathbb{R}$. For parameters $u \in(0,1)$ and $t \in[0,1]$, the following is a measurable function from $C[0,1] \times C[0,1] \times C[0,1] \times \ldots$ into $\mathbb{R} \cup\{-\infty, \infty\}$ (see Definition 2.13(b)):

$$
q_{u, t}(x):=\inf \left\{r \in \mathbb{R}: \quad z_{r, t}(x) \geq u\right\} .
$$

Note that for a given $t \in[0,1]$ and a given $x \in C[0,1] \times C[0,1] \times$ $C[0,1] \times \ldots, \quad q_{u, t}(x)$ is nondecreasing as $u$ increases in $(0,1)$.

For a given $t \in \mathbb{Q}_{[0,1]}$, the following is a measurable function from $C[0,1] \times$ $C[0,1] \times C[0,1] \times \ldots \rightarrow \mathbb{R}:$

$$
\psi_{t}(x):=\left\{\begin{aligned}
5 \cdot \int_{.4}^{.6} q_{u, t}(x) d u=\lim _{n \rightarrow \infty}\left[\frac{1}{n} \sum_{j=0}^{n-1} q_{.4+(j / n)(.2), t}(x)\right] \\
\\
\text { if }-\infty<q_{u, t}(x)<\infty \quad \forall u \in(0,1) \\
\text { otherwise. }
\end{aligned}\right.
$$

(See Remark 2.16.) Finally, define the function $f(x):=\left(f_{t}(x), t \in[0,1]\right)$ from $C[0,1] \times C[0,1] \times C[0,1] \times \ldots \rightarrow C[0,1]$ as follows:

For a given $x$, if $\left(\psi_{t}(x), t \in \mathbb{Q}_{[0,1]}\right)$ is a uniformly continuous function of $t \in \mathbb{Q}_{[0,1]}$, then $f(x)$ is the unique element of $C[0,1]$ such that $f_{t}(x)=$ $\psi_{t}(x) \forall t \in \mathbb{Q}_{[0,1]}$. Otherwise define $f(x):=0$, the zero element of $C[0,1]$. By an elementary argument, this function $f$ is measurable.

Step 2.26. Proof of (2.33). Let $k \in \mathbb{Z}$ be arbitrary but fixed.

Suppose $\omega \in \Omega$, and one denotes the sequence $x:=\left(X_{k}(\omega), X_{k+1}(\omega)\right.$, $\left.X_{k+2}(\omega), \ldots\right)$ of elements of $C[0,1]$. Then it is easy to check that (for $h \geq$ $0, n \geq 1, r \in \mathbb{R}, t \in[0,1], u \in(0,1))$

$$
I(S(k, k+h)(t) \leq r)(\omega)=\quad I_{h+1, r, t}(x)
$$




$$
\begin{aligned}
V(k, n, r, t)(\omega) & =v_{n, r, t}(x), \\
Z(k, r, t)(\omega) & =z_{r, t}(x), \quad \text { and } \\
Q(k, u, t)(\omega) & =q_{u, t}(x) .
\end{aligned}
$$

(See (2.3), (2.9), and (2.14).) For $t \in \mathbb{Q}_{[0,1]}$ one has that

$$
W(k, t)(\omega)=\psi_{t}(x)
$$

by (2.20). Finally, by Definition 2.23 ,

$$
Y_{k}(\omega)=f(x)=f\left(X_{k}(\omega), X_{k+1}(\omega), X_{k+2}(\omega), \ldots\right) .
$$

That is, (2.33) holds. This completes the proof of Theorem 1.1 for $C[0,1]$ valued random variables.

\section{Proof of Theorem 1.1 (for a general separable real Banach space).}

Before we begin the proof of Theorem 1.1 for general separable real Banach spaces, we need to get a couple of technical lemmas out of the way. Suppose $(S, d)$ is a complete separable metric space, and $x:=\left(x_{1}, x_{2}, x_{3}, \ldots\right)$ is a sequence of elements of $S$. We shall denote $T x:=\left(x_{2}, x_{3}, x_{4}, \ldots\right)$, the sequence obtained from $x$ by deleting the first entry. Also, the term "accumulation point of the sequence $x$ " will simply mean an element $s \in S$ (if such an $s$ exists) which satisfies $s=\lim _{m \rightarrow \infty} x_{k(m)}$ for some strictly increasing subsequence $k(1), k(2), k(3), \ldots$ of the positive integers.

Lemma 3.1. $\quad$ Suppose $(S, d)$ is a complete separable metric space, and $A$ is a compact subset of $S$. Then there exists a measurable function $f$ : $A \times A \times A \times \ldots \rightarrow A$ such that for every sequence $x:=\left(x_{1}, x_{2}, x_{3}, \ldots\right)$ of elements of $A$, one has that (i) $f(x)$ is an accumulation point of the sequence $x$, and (ii) $f(T x)=f(x)$.

Proof. Here we shall just give the definition of the function $f$. The verification of its stated properties (measurability and properties (i) and (ii)) is elementary and is left to the reader.

First, for each positive integer $n$ let $\left(a_{1}^{(n)}, a_{2}^{(n)}, \ldots, a_{m(n)}^{(n)}\right)$ be a finite sequence of distinct elements of $A$ such that $\forall s \in A, \exists k \in\{1,2, \ldots, m(n)\}$ such that $d\left(s, a_{k}^{(n)}\right) \leq 2^{-n}$.

An elementary observation will be needed. For $s \in A$ and $\varepsilon>0$, let $\bar{B}(s, \varepsilon)$ denote the closed ball $\subset A$ with center $s$, radius $\varepsilon$. Then for any $n \geq 2$, any $\ell \in\{1,2, \ldots, m(n-1)\}$, one has that $\bar{B}\left(a_{\ell}^{(n-1)}, 2^{-(n-1)}\right) \subset$ 
$\bigcup_{j} \bar{B}\left(a_{j}^{(n)}, 2^{-n}\right)$, where the union is taken over all $j \in\{1,2, \ldots, m(n)\}$ such that $d\left(a_{\ell}^{(n-1)}, a_{j}^{(n)}\right) \leq 3 \cdot 2^{-n}$. Hence, for any $n \geq 2$, any $\ell \in\{1,2, \ldots$, $m(n-1)\}$, for a given sequence $x:=\left(x_{1}, x_{2}, x_{3}, \ldots\right)$ of elements of $A$, if $d\left(x_{k}, a_{\ell}^{(n-1)}\right) \leq 2^{-(n-1)}$ for infinitely many $k \geq 1$, then there exists $j \in$ $\{1,2, \ldots, m(n)\}$ such that $d\left(a_{\ell}^{(n-1)}, a_{j}^{(n)}\right) \leq 3 \cdot 2^{-n}$ and $d\left(x_{k}, a_{j}^{(n)}\right) \leq 2^{-n}$ for infinitely many $k \geq 1$.

Now let us define the function $f$. Suppose $x:=\left(x_{1}, x_{2}, x_{3}, \ldots\right)$ is any sequence of elements of $A$. Let $J(1)(x)$ denote the least element of $\{1,2, \ldots$, $m(1)\}$ such that $d\left(x_{k}, a_{J(1)(x)}^{(1)}\right) \leq 2^{-1}$ for infinitely many $k \geq 1$. Now if $L \geq 2$ is an integer, then assuming the positive integers $J(1)(x), \ldots, J(L-$ $1)(x)$ have already been defined, let $J(L)(x)$ denote the least element of $\{1,2, \ldots, m(L)\}$ such that $d\left(a_{J(L-1)(x)}^{(L-1)}, a_{J(L)(x)}^{(L)}\right) \leq 3 \cdot 2^{-L}$ and $d\left(x_{k}, a_{J(L)(x)}^{(L)}\right) \leq 2^{-L}$ for infinitely many $k \geq 1$. Finally define $f(x)$ by $f(x):=\lim _{L \rightarrow \infty} a_{J(L)(x)}^{(L)}$.

As indicated above, the rest of the proof of this lemma is left to the reader.

Lemma 3.2. $\quad$ Suppose $(S, d)$ is a complete separable metric space, and $A_{1}, A_{2}, A_{3}, \ldots$ is a sequence of compact subsets of $S$. Then there exists a measurable function $h: S \times S \times S \times \ldots \rightarrow S$ with the following property: If $x:=\left(x_{1}, x_{2}, x_{3}, \ldots\right)$ is any sequence of elements of $S$ such that for some $n \geq 1, x_{k} \in A_{n}$ for infinitely many $k \geq 1$, then (i) $h(x)$ is an accumulation point of the sequence $x$, and (ii) $h(T x)=h(x)$.

Proof. We shall just give the definition of the function $h$. The proof of its stated properties will be left to the reader.

First, applying Lemma 3.1, for each positive integer $n$ let $f_{n}: A_{n} \times$ $A_{n} \times A_{n} \times \ldots \rightarrow A_{n}$ be a measurable function such that for every sequence $y:=\left(y_{1}, y_{2}, y_{3}, \ldots\right)$ of elements of $A_{n}, f_{n}(y)$ is an accumulation point of the sequence $y$, and $f_{n}(T y)=f_{n}(y)$.

Also, let $s_{0}$ be an element of $S$.

Now let us define the function $f$. Suppose $x:=\left(x_{1}, x_{2}, x_{3}, \ldots\right)$ is a sequence of elements of $S$. We shall define $f(x)$.

First, if there does not exist $n \geq 1$ such that $x_{k} \in A_{n}$ for infinitely many $k \geq 1$, then define $h(x):=s_{0}$.

Now suppose instead that there does exist such an $n$. Let $N(x)$ denote the least positive integer such that $x_{k} \in A_{N(x)}$ for infinitely many $k \geq 1$. Let $k(1)(x), k(2)(x), k(3)(x), \ldots$ be the strictly increasing sequence 
of all positive integers $k$ such that $x_{k} \in A_{N(x)}$. Finally, define $h(x):=$ $f_{N(x)}\left(x_{k(1)(x)}, x_{k(2)(x)}, x_{k(3)(x)}, \ldots\right)$.

This completes the definition of the function $h$. The rest of the proof of this lemma is left to the reader.

Now we are ready to begin the proof of Theorem 1.1.

Proof of Theorem 1.1. Suppose $B$ is a separable real Banach space. Suppose $\left(X_{k}, k \in \mathbb{Z}\right)$ is a sequence of $B$-valued random variables on a probability space $(\Omega, \mathcal{F}, P)$, and this sequence satisfies the hypothesis of Theorem 1.1. Without loss of generality, we assume that for every $k \in \mathbb{Z}$, every $\omega \in \Omega, X_{k}(\omega)$ is defined as an element of $B$.

It is well known (see e.g. van Dulst [5, p. 14] or Lindenstrauss and Tzafriri [9, p. 153]) that every separable real Banach space is isometric to some subspace of $C[0,1]$. Hence by a simple argument, we may (and do) assume without loss of generality that $B$ itself is a subspace of $C[0,1]$.

Definition 3.3. Applying Theorem 1.1 in the special case of $C[0,1]$ valued random variables (the special case proved in Section 2), let $g$ : $C[0,1] \times C[0,1] \times C[0,1] \times \ldots \rightarrow C[0,1]$ be a measurable function such that, defining for each $k \in \mathbb{Z}$ the $C[0,1]$-valued random variable $Z_{k}:=$ $g\left(X_{k}, X_{k+1}, X_{k+2}, \ldots\right)$, one has that

$$
\forall k \in \mathbb{Z}, \quad X_{k}=Z_{k}-Z_{k+1} \quad \text { a.s. }
$$

The random variables $Z_{k}$ defined here need not take their values in $B$. There is still some work left.

For each $k=1,2,3, \ldots, Z_{0}-Z_{k}=X_{0}+\ldots+X_{k-1}$ a.s. by (3.1). Hence by the hypothesis of Theorem 1.1, the family of distributions of the random variables $\left(Z_{0}-Z_{k}, k=1,2,3, \ldots\right)$ is tight. Also, the distribution of the random variable $Z_{0}$ itself is tight (a well known fact that can be found e.g. in Billingsley [2, p. 10, Theorem 1.4]). Hence by an elementary argument, the family of distributions of the random variables $\left(Z_{1}, Z_{2}, Z_{3}, \ldots\right)$ is tight.

Definition 3.4. For each positive integer $n$, let $A_{n}$ be a compact subset of $C[0,1]$ such that

$$
\forall k \geq 1, \quad P\left(Z_{k} \in A_{n}\right) \geq 1-2^{-n} .
$$

These particular sets $A_{1}, A_{2}, A_{3}, \ldots$ will play a key role in the next definition. 


\section{Definition 3.5.}

(A) For any given sequence $x:=\left(x_{1}, x_{2}, x_{3}, \ldots\right)$ of elements of $C[0,1]$, denote $T^{0} x:=x, T^{1} x:=T x$, and for $j \geq 2, T^{j} x:=\left(x_{j+1}, x_{j+2}, x_{j+3}, \ldots\right)$, the $j^{\text {th }}$ iteration of $T$ on $x$.

(B) Applying Lemma 3.2, let $h: C[0,1] \times C[0,1] \times C[0,1] \times \ldots \rightarrow C[0,1]$ be a measurable function such that if $x:=\left(x_{1}, x_{2}, x_{3}, \ldots\right)$ is a sequence of elements of $C[0,1]$ such that for some $n \geq 1, x_{k} \in A_{n}$ for infinitely many $k$, then (i) $h(x)$ is an accumulation point of the sequence $x$, and (ii) $h(T x)=h(x)$.

(C) If $x:=\left(x_{1}, x_{2}, x_{3}, \ldots\right)$ is a sequence of elements of $C[0,1]$, then $x$ will be said to satisfy "Condition C" if both of the following two statements hold:

(a) $\quad x_{j}=g\left(T^{j-1} x\right)-g\left(T^{j} x\right)$ for every $j=1,2,3, \ldots$ (where $g$ is the function in Definition 3.3), and

(b) there exists a positive integer $n$ such that $g\left(T^{j} x\right) \in A_{n}$ for infinitely many $j \geq 1$.

(D) Define the function $f: C[0,1] \times C[0,1] \times C[0,1] \times \ldots \rightarrow C[0,1]$ as follows:

$$
f(x):=g(x)-h\left(g(x), g(T x), g\left(T^{2} x\right), g\left(T^{3} x\right), \ldots\right)
$$

if $x$ satisfies Condition C; otherwise, $f(x):=0$ (the zero element of $C[0,1])$.

In the next lemma, we shall use the term "standard $\sigma$-field" from the very first paragraph of Section 1.

\section{Lemma 3.6.}

(1) The function $f$ is measurable (with respect to the standard $\sigma$-field on $C[0,1])$.

(2) For every sequence $x:=\left(x_{1}, x_{2}, x_{3}, \ldots\right)$ of elements of $B$, one has that $f(x) \in B$.

(3) The function $f$ restricted to $B \times B \times B \times \ldots$ is measurable with respect to the standard $\sigma$-field on $B$.

Proof. The proof of (1) is an elementary exercise involving just basic measuretheoretic techniques. This is also true of the proof of (3), once (2) is proved. We shall just give the proof of (2), and leave the proofs of (1) and (3) to the reader.

Suppose $x:=\left(x_{1}, x_{2}, x_{3}, \ldots\right)$ is a sequence of elements of $B$. Our task is to show that $f(x) \in B$. 
If $x$ fails to satisfy Condition C, then by Definition 3.5(D), $f(x)=0$ (the zero element of $C[0,1])$, which is of course an element of $B$.

Now suppose instead that the sequence $x$ does satisfy Condition C. Again refer to Definition 3.5. The element $h\left(g(x), g(T x), g\left(T^{2} x\right), \ldots\right)$ is an accumlation point of the sequence $\left(g(x), g(T x), g\left(T^{2} x\right), \ldots\right)$. Hence $f(x)$ is an accumulation point of the sequence

$$
\left(g(x)-g(T x), g(x)-g\left(T^{2} x\right), g(x)-g\left(T^{3} x\right), \ldots\right) .
$$

Also, for each $j \geq 1$,

$$
g(x)-g\left(T^{j} x\right)=\sum_{k=1}^{j}\left[g\left(T^{k-1} x\right)-g\left(T^{k} x\right)\right]=\sum_{k=1}^{j} x_{k},
$$

which is an element of $B$. It follows that $f(x)$ is an element of $B$. This completes the proof of part (2) of this lemma.

Definition 3.7. Referring to Lemma 3.6(2)(3), for each $k \in \mathbb{Z}$ define the $B$-valued random variable

$$
Y_{k}:=f\left(X_{k}, X_{k+1}, X_{k+2}, \ldots\right) .
$$

The task that remains is to prove that

$$
\forall k \in \mathbb{Z}, X_{k}=Y_{k}-Y_{k+1} \text { a.s. }
$$

Let us return to the compact sets $A_{1}, A_{2}, A_{3}, \ldots \subset C[0,1]$ in Definition 3.4. For each $n \geq 1$, each $j \geq 1$, define the event $D(n, j):=\left\{Z_{k} \notin A_{n} \quad \forall k \geq\right.$ $j$ \}. For each $n \geq 1$, one has that $D(n, 1) \subset D(n, 2) \subset D(n, 3) \subset \ldots$, and hence

$$
P\left(\bigcup_{j=1}^{n} D(n, j)\right)=\lim _{j \rightarrow \infty} P(D(n, j)) \leq \limsup _{j \rightarrow \infty} P\left(Z_{j} \notin A_{n}\right) \leq 2^{-n}
$$

by (3.2). That is, for each $n \geq 1$,

$$
P\left(Z_{k} \in A_{n} \text { for infinitely many } k \geq 1\right) \geq 1-2^{-n} \text {. }
$$

Definition 3.8. Let $\Omega^{*}$ denote the set of all $\omega \in \Omega$ such that (i) there exists $n \geq 1$ such that $Z_{k}(\omega) \in A_{n}$ for infinitely many $k \geq 1$, and (ii) $X_{k}(\omega)=Z_{k}(\omega)-Z_{k+1}(\omega)$ for all $k \in \mathbb{Z}$. 
As an elementary consequence of (3.1) and (3.5), one has that $P\left(\Omega^{*}\right)=1$.

Let $k \in \mathbb{Z}$ and $\omega \in \Omega^{*}$ be arbitrary but fixed. To prove (3.4), and thereby complete the proof of Theorem 1.1, it suffices to prove for this fixed pair $k, \omega$ that

$$
X_{k}(\omega)=Y_{k}(\omega)-Y_{k+1}(\omega) .
$$

Let $x$ denote the sequence of elements of $B$ defined by

$$
x:=\left(X_{k}(\omega), X_{k+1}(\omega), X_{k+2}(\omega), \ldots\right) .
$$

In the notations of Definitions 3.3 and 3.5 we have $x_{1}:=X_{k}(\omega), x_{2}:=$ $X_{k+1}(\omega), x_{3}:=X_{k+2}(\omega)$, and so on; and $g(x)=Z_{k}(\omega), g(T x)=Z_{k+1}(\omega)$, $g\left(T^{2} x\right)=Z_{k+2}(\omega)$, and so on.

By Definition 3.8, one has that $x_{j}=g\left(T^{j-1} x\right)-g\left(T^{j} x\right)$ for all $j \geq 1$. Also by Definition 3.8, $\exists n \geq 1$ such that $g\left(T^{j} x\right) \in A_{n}$ for infinitely many $j \geq 1$. Thus the sequence $x$ satisfies Condition $\mathrm{C}$ in Definition 3.5. Hence by (3.3), (3.7), and Definition 3.5(D),

$$
Y_{k}(\omega)=g(x)-h\left(g(x), g(T x), g\left(T^{2} x\right), \ldots\right) .
$$

By the same argument with the integer $k$ replaced by $k+1$, one has that

$$
Y_{k+1}(\omega)=g(T x)-h\left(g(T x), g\left(T^{2} x\right), g\left(T^{3} x\right), \ldots\right)
$$

(where $x$ is as defined in (3.7)).

Now by Definition 3.5(B),

$$
h\left(g(x), g(T x), g\left(T^{2} x\right), \ldots\right)=h\left(g(T x), g\left(T^{2} x\right), g\left(T^{3} x\right), \ldots\right) .
$$

Hence by (3.8), (3.9), and Definition 3.8,

$$
Y_{k}(\omega)-Y_{k+1}(\omega)=g(x)-g(T x)=Z_{k}(\omega)-Z_{k+1}(\omega)=X_{k}(\omega) .
$$

Thus (3.6) holds. This completes the proof of Theorem 1.1.

\section{References}

[1] I. Berkes, An extension of the Komlós subsequence theorem, Acta. Math. Hungar., 55 (1990), 103-110.

[2] P. Billingsley. Convergence of Probability Measures, Wiley, New York, 1968.

[3] R.C. Bradley, On a theorem of K. Schmidt, Statist. Probab. Letters, 24 (1995), 9-12. 
[4] - A "multiplicative coboundary" theorem for some sequences of random matrices, J. Theor. Probab., 9 (1996), 659-678.

[5] D. van Dulst, Reflexive and Superreflexive Banach Spaces, Mathematisch Centrum, Amsterdam, 1978.

[6] M.I. Gordin, On the behavior of the variances of sums of random variables forming a stationary process, Theory Probab. Appl., 16 (1971), 474-484.

[7] I.A. Ibragimov and Yu.V. Linnik, Independent and Stationary Sequences of Random Variables, Wolters-Noordhoff, Groningen, 1971.

[8] V.P. Leonov, On the dispersion of time averages of a stationary random process, Theory Probab. Appl., 6 (1961), 93-101.

[9] J. Lindenstrauss and L. Tzafriri, Classical Banach Spaces, Lecture Notes in Mathematics, no. 338. Springer-Verlag, New York, 1971.

[10] K. Schmidt, Cocycles on Ergodic Transformation Groups, Macmillan India, Delhi, 1977.

Received August 2, 1995. This research was partially supported by NSF grant DMS9500307.

INDIANA UNIVERSITY

Bloomington, IN 47405

E-mail address: bradleyr@ucs.indiana.edu 